\title{
In memoriam Maurice Theytaz
}

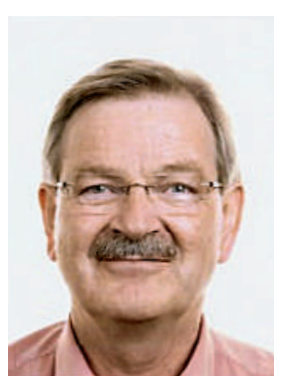

Dr Maurice Theytaz 17.3.1949-29.12.2014
Fin décembre 2014 nous quitta dans l'intimité de sa famille et sans faire de bruit le Docteur Maurice Theytaz, agé de 65 ans, après une courte maladie mais lourde, inattendue, alors qu'il avait mis sur pied tant de projets pour vivre une retraite aussi active que l'avait été toute sa vie.

Du point de vue professionnel, l'engagement de Maurice au sein de l'AI en faveur d'une meilleure réintégration et réadaptation des patients a été impressionnant, pendant plus de 30 ans. Suite à la création des "Services Médicaux Régionaux» (SMR), il a accepté le poste de médecin responsable du SMR Rhône en 2005, tout en gardant son cabinet de médecin de famille à temps partiel à Grimisuat. Au sein du SMR, il réussit à créer une équipe harmonieuse, créative, engagée en valorisant les différentes forces et qualités des médecins SMR y travaillant. Ses soucis constants étaient de simplifier les procédures administratives, de favoriser le contact direct et informel avec les médecins-traitants et les différents partenaires ainsi que le maintien de l'autonomie de l'appréciation médicale malgré les pressions financières et juridiques croissantes. Il ne se lassait pas de nous répéter: "Vous êtes médecins d'abord!» Pour des cas complexes, Maurice favorisait des décisions de bon sens, prises en concertation avec les collègues concernés de la réadaptation, les juristes et les gestionnaires.

Il nous a appris à appliquer nos connaissances dans un cadre législatif de plus en plus strict, tout en gardant le respect de notre déontologie médicale au-dessus des réflexions sur des statistiques et des quotas de rentes à atteindre. Malheureusement, parce que la corporation médicale n'a pas su acquérir et défendre suffisamment tôt de façon cohérente et concertée les bases et les prérequis de l'attestation de l'incapacité de travail, le législateur a pris de plus en plus de décisions d'ordre formatif qui limitent la marge d'appréciation et du bon sens médical et tend à dégrader les médecins assécurologiques en de simples fonctionnaires, sans aucune capacité de jugement. Avec d'autres collègues pionniers, Dr Theytaz a reconnu très tôt cette évolution inquiétante. Pour créer un contrepoids, il a beaucoup soutenu l'implémentation du travail de la SIM (Swiss Insurance Medicine) en Suisse romande. Son but était de bâtir des bases solides pour défendre une médecine assé- curologique cohérente, au service de nos patients et de notre société. Dr Theytaz s'est beaucoup investi au niveau suisse pour améliorer et standardiser les formations pour les médecins SMR. Il était en même temps convaincu qu'il était important d'avoir au sein du SMR des médecins qui continuaient à avoir une activité clinique leur permettant de rester en contact avec la réalité du terrain. L'engagement de Maurice dans un domaine médical décrié et attaqué par nombreux de nos collègues - mais aussi parfois en conflit de pouvoir avec les instances administratives et juridiques - a été sincère, loyal et cohérent tout au long de sa carrière. A côté de son engagement pour l'AI, il est resté un vrai médecin de famille engagé pour ses patients jusqu'au bout.

Pour des cas complexes, Maurice favorisait des décisions de bon sens, prises en concertation avec les collègues concernés.

Malheureusement, les structures du «new public management", les soi-disants "contrôles de qualité et de productivité» ainsi que des conflits interpersonnels ont eu raison de ses ambitions sincères et ont amené un changement délétère au sein du SMR Rhône. Il s'en est suivi un grand remaniement et de nombreux départs, dont celui de Maurice en 2014 Peu après ces turbulences, une maladie grave se déclara, complètement inattendue, qui devait l'emporter en quelques mois. Nous tenons à remercier tous ceux et celles qui ont soutenu Maurice dans ses derniers moments difficiles.

Il nous reste à dire un grand merci à Maurice au nom de tous ceux qui ont connu et apprécié son engagement et au nom de tous ceux qu'il a formé et encouragé dans leur parcours professionnel en leur donnant la passion de l'évaluation de l'incapacité de travail dans le respect de la déontologie médicale. Nous garderons en nous le souvenir d'une personnalité sincère, humble, au service de ses convictions, et son amitié laissera des traces indélébiles dans nos vies et nos cœurs. A sa femme Marie-Thérèse ainsi qu'à toute sa famille, nous présentons nos sincères condoléances au nom de notre corporation.

Dr Monique Lehky Hagen, ancien médecin SMR, présidente de la SMV 